\title{
CULTURA POLITECNICA, ENERGIA, INDUSTRIALIZZAZIONE
}

\author{
ANDREA SILVESTRI $(*)$
}

SunTO. - L'Esposizione nazionale di Milano del 1881, se confrontata sia con la precedente di Firenze nel 1861, sia con la partecipazione italiana a intermedie esposizioni internazionali, mostrava come finalmente avesse compimento anche da noi la prima rivoluzione industriale, quella sintetizzabile con il trinomio carbone-ferro-vapore. $\mathrm{Ma}$ Giuseppe Colombo del Politecnico di Milano, che aveva presentato Firenze $1861 \mathrm{su}$ "La Perseveranza" ed era ora tra gli organizzatori di Milano 1881, sapeva bene che era alle porte la seconda rivoluzione industriale, quella soprattutto dell'elettricità. Colombo lo sapeva bene perché partecipe di una pressoché contemporanea, questa sì rivoluzionaria, Esposizione internazionale di elettricità a Parigi, dove Edison metteva in mostra in Europa il sistema interamente di sua concezione per produzione, trasporto a distanza e utilizzazione (a scopo di illuminazione anche a incandescenza) dell'energia elettrica in corrente continua. Colombo, tornato a Milano, avviò la fondazione di quella che sarebbe stata la principale società elettrocommerciale italiana, la Edison, e (dopo un viaggio da Edison in America) realizzò nel 1883 la prima centrale elettrica europea, Santa Radegonda, per l'illuminazione del centro storico. Di lì a poco lo sfruttamento idroelettrico e il trasporto a distanza in corrente alternata avrà compimento con la centrale di Paderno d'Adda e con la trasmissione Paderno-Milano, non solo per illuminazione ma anche per forza motrice in ambito industriale e nei trasporti pubblici. Di qui in avanti l'elettrificazione del Paese ne accompagna la modernizzazione: nel frattempo la nascita al Politecnico dell'Istituzione Elettrotecnica Carlo Erba rafforza la formazione di nuovi tecnici, rappresentati da personaggi della statura di Giacinto Motta o Ettore Conti, mentre altri (per es. Giovanni Battista Pirelli o Alberto Riva) incontrarono successi internazionali nella produzione di cavi o macchinari elettrici.

$$
* * *
$$

ABSTRACT. - The Milan National Exhibition in 1881, if compared with the previous one in Florence (1861) and with the intermediate international exhibitions, shows that also Italy had finally completed the First Industrial Revolution, characterized by the trinomial

(*) Istituto Lombardo, Politecnico di Milano, Italia.

E-mail: andrea.silvestri@polimi.it 
coal-iron-steam. Giuseppe Colombo of the Politecnico di Milano, who had presented Florence Exhibition in 1861 in the newspaper "La Perseveranza" and subsequently was one of the promoter of the Milan National Exhibition in 1881, knew very well that the second Industrial Revolution - in particular for electricity - was about starting. He was aware because he took part to the revolutionary international Exposition of Electricity in Paris (1881), where Edison showed his new continue corrent system for production, longdistance transmission and distribution of electricity for incandescent lighting. When he came back to Milan, Colombo founded the most important electric company in Italy, Edison, and in 1883 (after one journey to find Edison in America), he devised the first European electrical power plant - Santa Radegonda - for the enlightenment of the old town centre of Milan. In few years the hydroelectric production and the long way distance alternating current transmission were achieved with the power plant of Paderno d'Adda and the electrical transmission line from Paderno to Milan, not only for enlightenment use but also for motive power, industrial use and public transport. Hereinafter the electrification of the Country Italy accompanied its modernization: the foundation of the Istituzione Elettrotecnica Carlo Erba in the Politecnico di Milano strengthened the training of new technicians, such as high profile personalities like Giacinto Motta or Ettore Conti, while entrepreneurs like Giovanni Battista Pirelli or Alberto Riva had international successes with the production of cables or electrical machines.

1. Le esposizioni, che dalla seconda metà del Settecento erano state, tanto più nel nostro Paese, di carattere prevalentemente agricolo ${ }^{1}$, con la Great Exhibition di Londra nel 1851 (Fig. 1) diventano davvero occasioni per sostenere l'innovazione tecnologica, diffonderne la conoscenza, e insieme trasmettere fiducia nel progresso mondiale (gli industrialisti, specie in Italia, avevano bisogno di legittimazione sociale, e, tra i destinatari, le classi lavoratrici dovevano trovare ragioni per attenuare la conflittualità). Questi modelli - persino le relative forme architettoniche, per esempio il Crystal Palace - diventeranno riferimenti, con costanti e varianti, anche per il futuro.

Dopo Londra 1851 partono analoghe iniziative nel continente, poi (da Philadelphia 1876, nel centenario della rivoluzione americana) anche negli Stati Uniti. In Italia la prima esposizione nazionale, subito nel 1861 a Firenze (Fig. 2), è un'occasione più politica che davvero tecnica, in un Paese arretrato ma desideroso e capace di misurarsi con la modernità: un reporter d'eccezione come Giuseppe Colombo, che stava per fondare con Francesco Brioschi il Politecnico, non sottace ai

1 Cfr. L. Aimone, Le esposizioni industriali a Torino (1829-1898), in Innovazione e modernizzazione in Italia fra Otto e Novecento, a cura di E. Decleva, C.G. Lacaita e A. Ventura, Milano, FrancoAngeli, 1995. 
lettori de "La Perseveranza" la «dolorosa insufficienza» della mostra, pur indicandola ottimisticamente quale «punto di partenza della storia industriale italiana» $»^{2}$. Analoga funzione di testimonianza, in un clima ancora da «grande "fiera di paese" »", ebbe la prima esposizione industriale di Milano nel 1871.

Le ragioni del ritardo italiano erano molte, e ricorderò per lo meno - accanto alla mancanza delle materie prime che qualificavano la prima rivoluzione industriale (carbone e ferro) - in epoca preunitaria il frazionamento politico in sette stati d'ancien régime, con frontiere fisiche ma anche culturali ${ }^{4}$, blocchi cioè alla libera espansione dei nuovi saperi: ciò penalizzava ulteriormente la nostra cultura tecnica, non molto curata né molto apprezzata, se a lungo si era mantenuta la situazione (persino nella più progredita Lombardia) che la professione dell'ingegnere fosse ancora elitaria e ereditaria, senza attenzione per l'istruzione non solo pratica e per il merito. Sotto questi punti di vista, grande rilievo avevano avuto le innovazioni del riformismo asburgico e la ventata davvero rivoluzionaria di Napoleone, che valorizzarono gli studi scientifico-tecnici e la loro fondamentale presenza nei curricula relativi'. Così l'Università di Pavia fu potenziata con strutture, docenti e discipline che tentavano di stare al passo con i tempi; e si istituivano o si rafforzavano organismi tecnici come il Corpo degli ingegneri di acque e strade destinato in Lombardia a importanti e durature realizzazioni. Altrove poi, come a Napoli, si avviò molto per tempo (1811) anche una Scuola di Ponti e strade, vero incunabolo delle future scuole politecniche italiane.

2. Nella Restaurazione, nei campi dell'istruzione tecnica pre-

2 Cfr. C.G. Lacaita, Giuseppe Colombo e le origini dell'Italia industriale, prefazione a Giuseppe Colombo, Industria e politica nella storia d'Italia. Scritti scelti: 1861-1916, a cura di C.G. Lacaita, Milano-Bari, Cariplo-Laterza, 1985, 20-21.

3 Cfr. G.M. Longoni, Dalla mostra del Sempione alla Fiera. Alcune riflessioni su Milano e le esposizioni, in Milano e l'Esposizione internazionale del 1906. La rappresentazione della modernità, a cura di P. Audenino, M.L. Betri, A. Gigli Marchetti, C.G. Lacaita, Milano, FrancoAngeli, 2008 (la cit. è a p. 12).

4 Di significati sia fisici sia metaforici del termine "frontiere" si è ragionato molto all'Istituto Lombardo una settimana prima di questo Convegno, durante la presentazione di Alberto Quadrio Curzio del volumetto di P. Bassetti, Svegliamoci Italici, Venezia, Marsilio, 2015.

5 A. Silvestri, Prima e dopo Napoleone. Università e professioni, in P. Del Negro, L. Pepe (a cura di), Le università napoleoniche. Uno spartiacque nella storia italiana ed europea dell'istruzione superiore, Bologna, Clueb, 2008. 
valse la continuità piuttosto che la rottura, ma a Milano la sospettosa e occhiuta vigilanza austriaca (ne sa qualcosa anche il nostro Istituto, tra ostacoli, lentezze, rinvii del Governo di Vienna) non favorì l'innovazione, che tuttavia ebbe momenti significativamente premonitori. Così, nel 1839 era nata a Milano la Società d'Incoraggiamento d'Arti e Mestieri (Fig. 3), per iniziativa dell'imprenditorialità aristocratica e borghese più attenta alle novità europee: quando l'impegno della Società cominciò a esprimersi attraverso i suoi corsi e i suoi laboratori (mentre le sue pubbliche dimostrazioni, affollate e apprezzatissime, sarebbero state aperte a un pubblico generalista), trovò avvio e poi realizzazione il processo di acculturamento tecnico di operai, capioperai, quadri intermedi, nella stessa direzione che avrebbe avuto compimento a livello superiore con il Politecnico: non a caso uno degli animatori della Società d'Incoraggiamento era e sarà a lungo proprio Colombo.

Quasi contemporaneamente, e quasi contemporaneamente con i primi Congressi nazionali degli scienziati, Carlo Cattaneo avviava la sua impresa della rivista "Il Politecnico", dove le «arti utili» avevano posto non subordinato, anzi, rispetto alle «arti belle», con attenzione alla cultura anche tecnica e imprenditoriale d'Oltralpe e alle potenzialità variegate ma spiccate dell'«incivilimento» del nostro Paese.

L'Istituto Lombardo, di fondazione napoleonica, aveva una tradizione scientifica che risaliva al suo primo presidente Alessandro Volta ma che si esplicava anche in premi periodici per "utili invenzioni", i cui prodotti, macchine modelli disegni, costituirono la base di un interessante Gabinetto tecnologico (Fig. 4). Pur nel clima poliziesco di cui dicevo, l'Istituto Lombardo nel 1848 studiò per incarico del governo - relatore lo stesso Cattaneo, già peraltro attivo nella Società d'Incoraggiamento - una riforma di tutti gli studi, compresi quelli universitari: quanto agli ingegneri e architetti il progetto prevedeva, sull'esempio europeo, un Politecnico specializzante a Milano da raccordarsi con la formazione a Pavia, formazione teorica e sempre meno capace (estranea com'era a un vero «movimento [...] industriale» ${ }^{6}$ ) di aggiornarsi e rinnovarsi. Le Cinque Giornate avrebbero travolto il progetto del Lombardo, ma quando il Piemonte annetterà la

6 Cfr. in C.G. Lacaita, A. Silvestri (a cura di), Francesco Brioschi e il suo tempo (1824-1897), vol. III, Scritti e discorsi (a cura di C.G. Lacaita), 32. 
Lombardia nel '59 e dedicherà uno dei suoi primi provvedimenti legislativi (la Legge Casati) all'unificazione dei due modelli di scuola, nella sua attuazione a Milano i semi del progetto del Lombardo non sono perduti ${ }^{7}$, nonostante l'avversione, per motivi ideologici e politici già dai tempi della rivoluzione del ' 48 , di Cattaneo nei confronti di Gabrio Casati.

Da lì a poco, 1863, sarebbe appunto nato (così come la Scuola di applicazione per gli Ingegneri a Torino) il Regio Istituto Tecnico Superiore di Milano, con il contributo fondamentale - oltreché del fondatore Brioschi - del cofondatore Colombo (Fig. 5). Il Politecnico di Milano avrebbe subito affiancato, nei suoi corsi di studio, alla sezione tradizionale degli ingegneri civili, alla scuola normale per formare insegnanti di discipline scientifiche ${ }^{8}$, una sezione meccanica, di lì a poco rinominata industriale - orientata ad affrontare la formazione dei nuovi ingegneri meccanici, ma poi anche dei nuovissimi ingegneri elettricisti, e dopo ancora degli ingegneri chimici. Nel frattempo, subito nel 1865, era partita la sezione degli ingegneri architetti, di cui pure ragionava il progetto del Lombardo e di Cattaneo, la quale avrebbe coinvolto in sinergia con il Politecnico l'Accademia di Belle Arti di Brera, auspice l'autorevolissimo Camillo

7 Cfr. A. Silvestri, Il progetto di riforma scolastica dell'Istituto Lombardo (1848, relatore Carlo Cattaneo) e la Legge Casati (1859), «Rendiconti», Milano, Istituto Lombardo Accademia di Scienze e Lettere, 145, 2012.

8 Equivalente, in ambito tecnico, alla preparazione degli insegnanti di discipline umanistiche fornita dalla coeva Accademia scientifico-letteraria, che diventerà infatti la Facoltà di lettere e filosofia dell'Università degli Studi di Milano (1924). Ma la Scuola normale del Politecnico ebbe vita stentata fin dal reale avvio nel 1867, poiché non dava laurea, e gli aspiranti insegnanti preferivano frequentare Ingegneria al Politecnico, o la Facoltà di Scienze fisiche, matematiche e naturali a Pavia. Invano il direttore del Museo Civico di Storia Naturale (sorto nel 1838) e docente di Geologia al Politecnico, Ettore Artini, sostenuto da Colombo, cercò di trasformare la prevalente sezione di Scienze in un'autonoma Facoltà di scienze naturali del Politecnico, la quale invece nascerà nel '24 con la Statale. Proprio in vista di questa novità, nel 1921, la sezione normale del Politecnico fu soppressa e l'annesso Orto botanico passò all'Istituto superiore di Agricoltura, già Scuola superiore di Agricoltura, nata nel 1870. Su tutto quel che precede cfr. E. Canadelli, Alla ricerca del coordinamento. Dal «grande Politecnico» all'«Università politecnica», in Milano scientifica 1875-1924, vol. 1, La rete del grande Politecnico, a cura di E. Canadelli, Milano, Sironi, 2008. E si ricorderà che anche la Bocconi avrebbe dovuto inizialmente sorgere, nel 1898, come sezione economica del "grande Politecnico". 
Boito, che avrebbe insegnato da ora in poi per decenni anche al Politecnico. Queste grandi novità, ben presto esportate in tutta l'Italia unita, avrebbero favorito la progressiva corsa del Paese verso l'innovazione tecnologica.

Circa le esposizioni, non mancherà la partecipazione italiana all'Esposizione internazionale di Londra nel 1862 e a quelle universali di Parigi (1867) e di Vienna (1873). Ma intanto all'Esposizione nazionale campionaria del 1871 a Torino, per l'apertura del Frejus (ecco un trait d'union con Milano 1906 per il Sempione), la sede dell'evento fu proprio quel Museo industriale di Torino, dalla cui fusione con la Scuola di Applicazione sarebbe nato nel 1906 il Politecnico di Torino: anche in questo caso le collezioni del Museo industriale (1862), secondo i famosi esempi parigino e londinese, erano state raccolte durante la partecipazione italiana alle esposizioni del passato (per esempio, il Regno di Sardegna aveva inviato «147 tecnici, operai e artigiani» a Londra nel 1851).

3. Veniamo finalmente alla seconda esposizione nazionale, Milano 1881 (Fig. 6), organizzata dallo stesso Colombo. Si trattava di una sorta di bilancio dei primi vent'anni del Regno d'Italia, e insieme della celebrazione del mito di Milano "capitale morale". La mostra si svolgeva nei giardini di Villa Reale, a pochi passi dalla sede che allora ospitava il Politecnico (il Palazzo della Canonica in piazza Cavour), in padiglioni di ferro e cristallo emuli di evoluti modelli europei. Alla Scala fu rappresentato il balletto Excelsior (Fig. 7), un'esaltazione ingenuamente ${ }^{10}$ positivistica della modernità e del progresso (il titolo, ormai desemantizzato, significa «più in alto»). Vi sono celebrati i successi del

9 Cfr. Aimone, Le esposizioni internazionali a Torino (1829-1898), cit., 520. Invece parla «di 12 operai del Granducato di Toscana [e] della presenza alla stessa esposizione di una comitiva di operai degli Stati Sardi» A. Pellegrino, Macchine come fate. Gli operai italiani alle esposizioni universali (1851-1911), Milano, Fondazione IsecGuerini e associati, 2011, 70. Il volume della Pellegrino si segnala per l'inquadramento generale delle esposizioni rispetto al tema del lavoro, in Italia con un legame particolare, se in occasione di quella Colombiana di Genova del 1892 (per il centenario della scoperta dell'America) si svolse il congresso fondativo del Partito Socialista Italiano, mentre quello della Confederazione Generale del Lavoro ebbe luogo a Milano in concomitanza con quella per il Sempione nel 1906.

10 Anche la struttura dell'illustrazione di Fig. 7 è ingenua, perché «la composizione è esattamente quella delle pale d'altare dopo la Controriforma, con in alto il cielo 
vapore e dell'elettricità, il taglio dell'istmo di Suez e il traforo del Cenisio, con la vittoria finale della Luce sull'oscurantismo. Molte le pubblicazioni celebrative di Milano 1881: uno di questi volumi, se per l'Esposizione universale di Parigi nel 1867 Victor Hugo aveva scritto l'introduzione della guida ufficiale, affiancava i nomi dei capiscuola veristi Capuana e Verga, siciliani ma in quegli anni milanesi d'adozione, con due scritti ricchi di riferimenti alle innovazioni tecniche della «città più città d'Italia» e dei suoi dintorni.

Colombo stesso commentava pubblicamente Milano 1881 anche a confronto con Firenze 1861; la «cresciuta cultura tecnica» e «quella schiera di giovani istruiti [nelle] nostre principali scuole d'applicazione» ${ }^{11}$ avevano fatto compiere passi in avanti, molti per qualità e quantità: l'industria meccanico-metallurgica si era sviluppata nei campi delle ferrovie e della navigazione, si erano potenziate e meccanizzate le imprese tessili, le macchine per l'agricoltura, l'attività manifatturiera grande e piccola. A proposito di industrializzazione, la preferenza di Colombo, per Milano, per ora (fino a quando lui stesso avvierà la Edison e cioè una sempre più grande industria elettrica) è per piccoli «lavoreri» di articoli di lusso che non richiedono troppa forza lavoro: forza lavoro sia in termini di energia necessaria (prevalentemente da macchine a vapore, per le esigue disponibilità idrauliche a Milano), sia in termini di lavoro umano per i rischi di grandi concentrazioni di pericolose masse operaie. E puntualmente, all'Esposizione, accanto alle gallerie delle macchine, delle carrozze, delle industrie estrattive, delle piccole industrie, ecc., si trovano padiglioni molto più tradizionali (la Galleria del lavoro della seta, con tante Lucia Mondella in vista, dell'Agricoltura, la Sala dei gioielli, della stampa e dei tessuti, dei mobili artistici, dell'oreficeria).

Colombo, mentre ricapitolava la strada compiuta finalmente anche da noi dalla prima rivoluzione industriale, non poteva ignorare quali grandi progressi poteva e anzi doveva riservare l'elettricità. Non lo ignorava perché dell'illuminazione ad arco, in mostra a Milano accanto a

stellato della Madonna, che trionfa» sui segni del nuovo e, ancora sotto, sul «palcoscenico [...] dei terrestri» (A.C. Quintavalle, Le figure ingannevoli, prefazione a I. Barzaghi, Milano 1881: tanto lusso e tanta folla. Rappresentazione della modernità e modernizzazione popolare, Milano, Silvana Editoriale, 2009, 13).

11 Cfr. negli Scritti scelti di Colombo, cit., 256. 
quella tradizionale a gas, era stato interprete tempestivo in conferenzeesibizioni di successo alla Società d'Incoraggiamento o altrove: anche se restava in lui (ma presto si sarebbe dissipato) qualche pregiudizio rispetto alle "esagerazioni americane" su quel genio tecnico ma anche commerciale-comunicativo che era Edison e sui suoi studi per la suddivisione della luce elettrica. Tuttavia la meta non era lontana: in futuro anche l'Italia sarebbe stata in grado di sfruttare le ricche disponibilità idriche per una generazione di grandi dimensioni, con la possibilità di trasmissioni di forza per via elettrica a grandi distanze inattuabili per via meccanica, e con l'illuminazione elettrica non solo concentrata in potenti fari ad arco (Fig. 8) ma suddivisa tra tante e modulabili lampade a incandescenza. Tutto questo retroterra di competenze di Colombo sulle prospettive delle applicazioni dell'elettricità trovava, quasi contemporaneamente a Milano 1881, solo pochi mesi dopo, un'occasione davvero rivoluzionaria a Parigi, all'Esposizione internazionale di elettricità (Fig. 9). Edison vi presentava il suo "sistema" elettrico, con componenti (tutti di sua progettazione e realizzazione) per la produzione, il trasporto, e l'utilizzazione dell'energia elettrica per uso di illuminazione. Era un sistema che finalmente somigliava come struttura a quello della dominante illuminazione a gas, sistema per generare energia elettrica in posizione centrale (donde in nome di centrale, da "stazione centrale", perché sede della produzione elettrica), sistema a rete per il trasporto a distanza, sistema per utilizzare l'elettricità anche nella nuova forma di illuminazione a incandescenza tramite lampade a filamento di carbone: sistema che sarebbe diventato economicamente conveniente con lo sviluppo dell' $\mathrm{i}$ droelettrico. La nuova illuminazione è nuova sia rispetto alle vecchie lampade ad arco, ma nuova soprattutto proprio rispetto al gas, il quale avrebbe cercato, oltreché di ridurre le sue tariffe di monopolista, di trovare per se stesso migliorie e aggiustamenti, senza impedire però che il nuovo trionfasse sul vecchio.

4. Tra i visitatori d'eccezione di Parigi 1881 figuravano Galileo Ferraris del Museo industriale di Torino (Museo che era nel frattempo diventato anche erogatore di corsi agli allievi ingegneri industriali) e Colombo stesso, il quale era già stato per esempio a Parigi '67, a Vienna '73, a Philadelphia '76, a Parigi '78 (poi sarà ancora a Parigi '89), e che nell'81 era nel comitato promotore della partecipazione italiana a Parigi. Colombo e Ferraris, rientrati in Italia, sarebbero diventati - ciascuno a suo modo, secondo la propria personale inclinazione - protagonisti del 
mondo elettrico. Colombo avrebbe fondato la Società Edison, e, dopo un viaggio a New York per conoscere Edison e acquisirne brevetti e collaboratori, sarebbe stato l'artefice della prima centrale elettrica sistema Edison in Italia (in realtà la prima in Europa). La centrale di Santa Radegonda (1883) era a carbone (nella prima delle immagini se ne scorge la ciminiera di fianco al Duomo), e in corrente continua a $110 \mathrm{~V}$ con un raggio di azione, perciò, dell'ordine al più del chilometro: era il percorso dell'illuminazione stradale e di negozi in Galleria, in Corso Vittorio Emanuele, in piazza del Duomo, in piazza e nel Teatro alla Scala. Le tecnologie di Santa Radegonda (Fig. 10) sarebbero state superate dopo non molti anni: infatti la cosiddetta battaglia dei sistemi tra la corrente alternata e la continua sarebbe stata inesorabilmente vinta dall'alternata, ben presto in forma non solo monofase o bifase ma pure trifase; e anche il combustibile carbone, d'importazione, avrebbe lasciato il posto al "carbone bianco", la grande disponibilità idraulica - nelle Alpi, negli Appennini, e via via anche al Sud - che Colombo e Ferraris da anni sognavano potesse essere non sciupata e sperperata, ma impiegata (oltreché per scopi irrigui) per trarne forza motrice. I due fattori tecnologici appena menzionati - la natura della corrente e la "houille blanche" - avrebbero risposto alle più moderne esigenze prima con la centrale idroelettrica di Tivoli e il trasporto in alternata Tivoli-Roma (1892), poi (davvero su larga scala) con la centrale di Paderno sull'Adda (1898) (Fig. 11) e con la trasmissione a distanza per via elettrica Paderno-Milano $(32 \mathrm{~km})$, all'allora elevatissima tensione di $12,5 \mathrm{kV}$ in corrente alternata trifase. E circa le utilizzazioni, queste non riguardavano solo l'illuminazione, ma anche la forza motrice, e non solo in ambito industriale ma anche nei trasporti pubblici. L'elettrificazione delle ferrovie sarà affrontata più tardi, all'inizio del nuovo secolo, e anche in questo caso a conclusione di un dibattito esterno e interno: esterno contro $\mathrm{i}$ sostenitori delle vecchie motrici a vapore, interno tra i fautori della trazione in continua oppure in alternata, monofase e trifase (per esempio, la trazione elettrica del Sempione sarà trifase ad alta tensione).

5. A Parigi 1881 Galileo Ferraris ${ }^{12}$ (Fig. 12) era il delegato governativo italiano alla mostra: da fisico tecnico di stampo tradizionale che

12 A. Silvestri (a cura di), Galileo Ferraris e l'AEI. Uomini e sodalizi della scienza elettrica, Milano, All'insegna del pesce d'oro di Vanni Scheiwiller, 1998. 
era (già famoso per le sue ricerche nel campo del vapore, delle applicazioni ottiche e acustiche, incluso il telefono), Ferraris diventerà un finissimo studioso di elettrotecnica ponendo le basi della disciplina in corrente alternata. Alla Mostra internazionale di Elettricità che organizzò a Torino nel 1884 (Fig. 13) - anche qui con una galleria del lavoro in ferro e vetro - $\mathrm{fu}$ annessa la trasmissione in corrente alternata a distanza tra Torino e Lanzo, $42 \mathrm{~km}$ a $3000 \mathrm{~V}$, a margine della quale Ferraris indagò, con profondità di metodo e ricchezza di risultati, il funzionamento della nuova e ancora misteriosa macchina che vi era impiegata: così misteriosa che era ancora chiamata "generatrice secondaria", essendo in realtà un rudimentale trasformatore. È proprio questo componente che solo in alternata consente nei siti di generazione di elevare la tensione, per trasmettere a distanza con correnti minori, con minori cadute di tensione e molto minori perdite, riabbassando poi la tensione verso le singole utilizzazioni. L'anno successivo, 1885, Ferraris avrebbe scoperto il principio del campo magnetico rotante, su cui si sarebbero basati i motori asincroni, più potenti e versatili di quelli in corrente continua, che avrebbero contribuito - con il trasformatore - a decretare la superiorità dell'alternata.

La contesa sulla priorità del campo magnetico rotante con il serbo naturalizzato americano Nikola Tesla (Fig. 14), anche lui grande elettricista della corrente alternata, non avrebbe coinvolto Ferraris, convinto positivisticamente che le scoperte della scienza dovessero essere messe a disposizione dell'umanità senza questioni di brevetti e privative. $\mathrm{Ma}$ all'Esposizione di Francoforte del 1891 - quella del grandioso esperimento in corrente alternata trifase di generazione, trasformazione, trasporto da Lauffen ai luoghi dell'Esposizione su $175 \mathrm{~km}$ a $15 \mathrm{kV}$, con utilizzazione mista per forza e luce - Ferraris sarebbe stato riconosciuto e acclamato pubblicamente come l'inventore del Ferrarischefeld. Il trionfo sarebbe continuato a Chicago nel 1893 (Fig. 15), dove accompagnava Ferraris il suo giovane allievo Camillo Olivetti, rimasto poi in America per documentarsi sulla relativa industrializzazione e per fare una personale esperienza di assistente universitario, prima del rientro in Italia e della fondazione della sua ditta di strumenti elettrici CGS (Centimetro Grammo Secondo) (Fig. 16) inizialmente a Ivrea e poi a Milano. Lo stimolo all'internazionalizzazione e all'innovazione tecnologica era la stessa secondo cui Colombo aveva indirizzato nel 1871 il suo neolaureato Giovanni Battista Pirelli, in viaggio-premio per l'Europa più evoluta, a concentrare la sua attenzione, piuttosto che sull'ormai 
collaudata industria serica, sulle nuove produzioni come quella del «caoutchouc».

6. Come ho detto, già a Milano 1881 la «magnifica fantastica illuminazione» della Galleria affiancava al gas le lampade elettriche ad arco, e Santa Radegonda illuminerà anche a incandescenza il centro cittadino; il trionfo dell'elettricità in giro per il mondo, tra gli ultimi decenni dell'Ottocento e i primi del Novecento, trova riscontro anche in Italia. Una tappa fondamentale, destinata a formare le future classi dirigenti tecniche e non solo in Lombardia e in Piemonte, è nel 1886'87 la nascita al Politecnico dell'Istituzione Elettrotecnica Carlo Erba, e a Torino, contemporaneamente, la Scuola superiore di elettrotecnica di Ferraris al Museo industriale ( $m a$, poco sfasati nel tempo, insegnamenti di Elettrotecnica, magari liberi, si istituiscono nelle Università di Roma e di Napoli) ${ }^{13}$. Via via alla Edison si affiancano altre società elettrocommerciali, talora a prevalente capitale Edison, come la Conti o l'Emiliana, oppure indipendenti ( $m$ a con crescenti interconnessioni fisiche e scambi commerciali tra le relative reti) come la Società Idroelettrica Piemonte (Sip), la Società Adriatica di Elettricità (Sade) nel Veneto, a Roma la Società Anglo-romana per il gas e l'elettricità, al sud la Società Meridionale di Elettricità (Sme); e poi, all'inizio del secolo, le aziende elettriche municipali (le più significative a Milano, a Torino, a Roma).

Dopo la crisi economica di fine secolo e, a Milano, dopo le cannonate di Bava Beccaris, era ripreso il processo di crescita (nelle attività industriali, agricole, commerciali-finanziarie, edilizie) di una città protesa verso l'Europa, con un'imprenditoria meno chiusa alle ragioni umanitarie e alle nuove istituzioni operaie e socialiste, che crede in quest'avanzata e ne agevola le finalità anche sociali. Nel 1906 il Sempione ne sarà simbolo e emblema.

7. L'idea, il progetto di un'Esposizione mondiale a Milano (Fig. 17) era in questo contesto ben pertinente, così come lo era - per celebrare l'apertura del Sempione - il tema iniziale dei mezzi di trasporto e di comunicazione: l'allargamento del tiro fino ad abbracciare atti-

13 Cfr. V. Cantoni, A. Silvestri (a cura di), Storia della tecnica elettrica, Milano, Cisalpino, 2009, 88-91. 
vità produttive, tecnologiche, di igiene e tutela del lavoro, di creatività artistica era rappresentativo di vitalità e modernità (beninteso non prive di contraddizioni). Per l'Esposizione internazionale di Milano 1906 il Politecnico era, con il Comune, la Camera di Commercio, ecc., tra gli Enti più coinvolti: i suoi personaggi eminenti portavano in sé le insegne di altre istituzioni a cui pure appartenevano, quasi tutti dell'Istituto Lombardo; Giuseppe Colombo significava anche la Edison o la Società d'Incoraggiamento; Cesare Saldini la Società Umanitaria; Giovanni Celoria l'Osservatorio di Brera; Angelo Salmoiraghi, dell'omonima industria ottica, gettava la luce del grande faro dall'Esposizione «fino alle alture di Bergamo, di Como, Varese ${ }^{14}$; Giovanni Battista Pirelli metteva a disposizione il suo padiglione all'Associazione Elettrotecnica Italiana (fondata nel '96-97 da Ferraris e Colombo) ${ }^{15}$ per esperimenti su cavi e linee aeree in altissima tensione, qualche centinaia di $\mathrm{kV}$, facendo uso di strumentazione, in particolare un trasformatore elevatore e un voltimetro elettrostatico, della CGS di Camillo Olivetti.

Per una mostra del genere occorreva una sede meno casalinga dei giardini di via Palestro, ed erano insufficienti anche gli spazi espositivi tra il Parco e l'Arena; ci si espandeva così fino alla Piazza d'Armi con il collegamento avveniristico - purtroppo effimero anch'esso, come tutte meno una (l'Acquario) le strutture in gesso e cartapesta della scenografica "Città Bianca" ${ }^{16}$ - di una ferrovia elettrica monofase sopraelevata lunga circa $1 \mathrm{~km}$ (Fig. 18), prefigurazione purtroppo, ripeto, simbolica (come del resto la fitta rete filoviaria elettrica in Piazza d'Armi, lunga circa $20 \mathrm{~km}$ ) di futuri collegamenti urbani e metropolitani.

Ma in tanto ottimismo, a Milano 1906, facevano riscontro i giganteschi cannoni Krupp esposti dalla Germania; la presenza di armi aveva caratterizzato anche le esposizioni precedenti, spesso camuffando le

14 Cfr. A. Silvestri, La comunicazione tecnico-scientifica sull'Esposizione: qualche campionatura, in Milano e l'Esposizione internazionale del 1906, cit., 75.

15 Cfr. A. Silvestri, Il centenario AEI e Galileo Ferraris, Milano, Associazione Elettrotecnica ed Elettronica Italiana, 1997.

16 Cfr. I. Barzaghi, Comunicazione per immagini e rappresentazione della modernità, in Milano e l'Esposizione internazionale del 1906, cit., 35. Della stessa autrice si segnala inoltre il già cit. volume Milano 1881: tanto lusso e tanta folla. Rappresentazione delle modernità e modernizzazione popolare. L'architettura di Expo 1906 è definita da Arturo Carlo Quintavalle già solo con il titolo Templi, cupole: un minestrone di stili esotici di un articoletto sul "Corriere della Sera", 26 marzo 2015. 
esigenze commerciali in coreografie quasi ludiche, ma ora non poteva non essere evocata la "Pace armata" ${ }^{17}$, con elementi di pressione sull'Italia a favore della Triplice contro i ravvicinamenti alla Francia, e con la tragica evocazione di un futuro di guerra non lontano.

8. Già prima della Grande Guerra era partito a Milano il progetto di Città Studi, non solo per una nuova sede del Politecnico in quanto protagonista della industrializzazione e anzi della modernizzazione del Paese, ma anche destinata a ospitare istituzioni di istruzione superiore varie che allargassero e segnassero il sostegno della città alla scienza e alla cultura. Durante il conflitto, quando pareva vicina la fine delle ostilità, e in vista di nuovi modelli nei futuri rapporti commerciali, circolarono ipotesi di una "fiera di campioni" 18 prevista più per gli operatori che per il grande pubblico. Le prime due edizioni della Fiera si svolsero in realtà nel 1920 e nel 1921 tra Porta Nuova e Porta Venezia, al risparmio, con solo 15 giorni di esposizione ma buon bilancio degli affari trattati. Talché partì la proposta sia di un ente autonomo con un proprio statuto, sia dell'acquisto dell'area della ex Piazza d'Armi: ciò che avvenne nel '22, occasione di una terza fiera svoltasi sia nei primi nuovi edifici realizzati a tempo di record sia in vecchi stand in legno che si diceva riutilizzassero - continuità tra passato e futuro - resti di baracche per i reduci di Caporetto ${ }^{19}$.

I tempi tuttavia stavano cambiando, il fascismo era alle porte, il tessuto universitario (che aveva già visto la nascita autonoma della Bocconi, 1902, e della Cattolica di padre Agostino Gemelli, 1921) stava per trasformarsi ancora: la creazione della Regia Università (la futura Statale), da parte del ginecologo già professore a Pavia Luigi Mangiagalli, rimetteva tra l'altro in discussione vecchi e faticosi equilibri (per es. tra Milano e Pavia). A livello nazionale la riforma Gentile del 1923 avrebbe tagliato finanziamenti alle Facoltà scientifiche - anche se per il Politecnico sarebbe intervenuta economicamente la Fondazione Politecnica Italiana voluta nel 1925 dalla Edison. Quanto

17 Cfr. P.L. Bassignana, La vetrina dell'industria germanica, in Milano e L'Esposizione internazionale del 1906, cit., p. 109.

18 Cfr. G.M. Longoni, Dalla Mostra del Sempione alla Fiera, in Milano e l'Esposizione internazionale del 1906, cit., p. 18.

19 Ibidem, p. 20. 
alla Fiera (Fig. 19), si consumava la rottura con il comune socialista, all'internazionalità si sostituiva il regionalismo, alle utopie di progresso e pace le celebrazioni di regime o le celebrazioni tout court.

Vorrei fermarmi qui, e chiudere con le parole quasi profetiche pronunciate per l'inaugurazione di Milano 1906 da Achille Manfredini, laureato politecnico e esponente del partito radicale, in uno degli editoriali della sua rivista "Il Monitore Tecnico". Manfredini sintetizza bene, ai tempi del Sempione (ma non è difficile l'estensione del ragionamento ai tempi della Fiera, anche se allora Manfredini era già morto), il filone dell'insofferenza $a^{20}$ per la pompa, l'ufficialità, la mondanità, e lo scontento - nonostante i grandi numeri di visitatori - per l'attenzione insufficiente ai lavoratori e alla questione sociale: già a Milano 1881 non erano mancati «gli stanchi delle esposizioni» (Luigi Luzzatti) ${ }^{21}$ né gli attacchi velenosi della pubblicistica satirica, ma i riti e i cliché della Fiera anche in età repubblicana avranno il sopravvento sugli ideali progressisti, pacifisti, sociali. E allora, ecco Manfredini: «rimasero pressoché del tutto estranei [...] i lavoratori», mentre gli interventi delle Autorità furono «non felici davvero, come limitati ad una ampollosa e retorica parafrasi del programma» $»^{22}$.

20 È del resto nota l'avversione alle Esposizioni di grandi personalità internazionali "di sinistra" come Marx, Engels, Benjamin. Così come sono note le critiche dell'«Avanti!» a Milano 1906.

21 Cfr. I. Piazzoni, Milano e le esposizioni universali (1860-1900), in Innovazione e modernizzazione in Italia, cit., 545.

22 Per Manfredini e le relative citazioni, si veda A. Silvestri, La comunicazione tecnico-scientifica sull'Esposizione, cit., in Milano e l'Esposizione internazionale del 1906, cit., 63. 


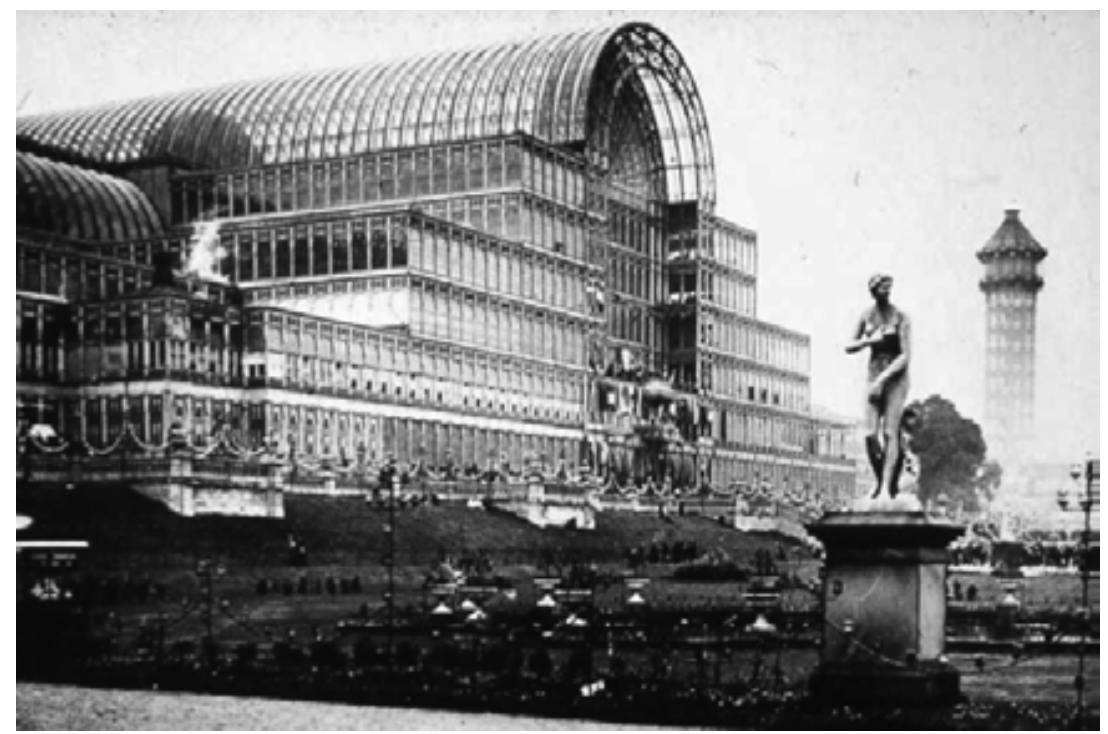

Fig. 1. Great Exbibition of the Works of Industry of all Nations, Londra, 1851.

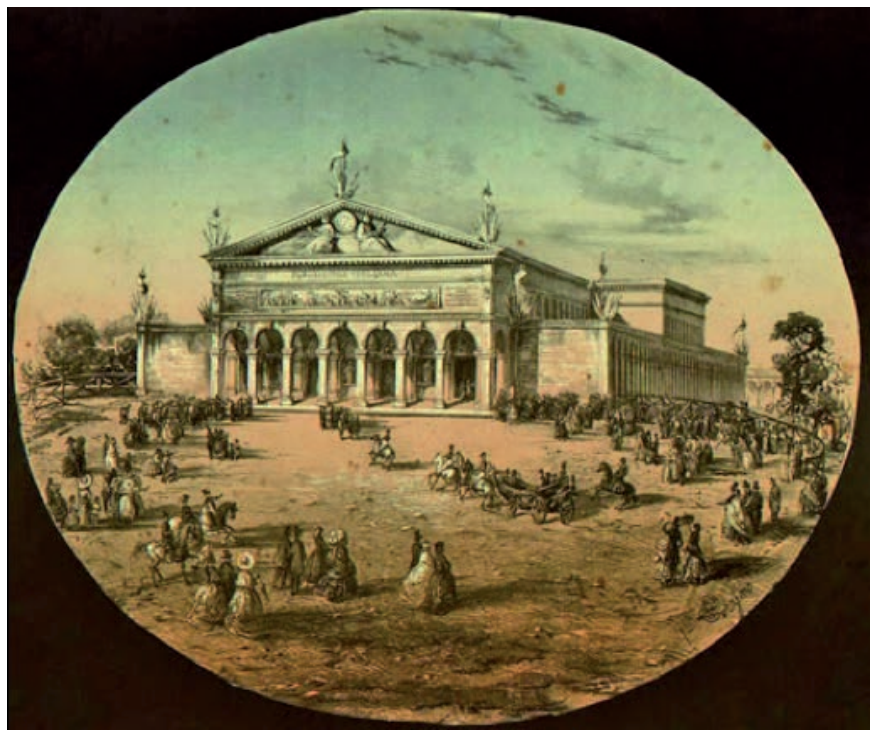

Fig. 2. Esposizione Nazionale di Prodotti Agricoli e Industriali e di Belle Arti, Firenze, 1861. 


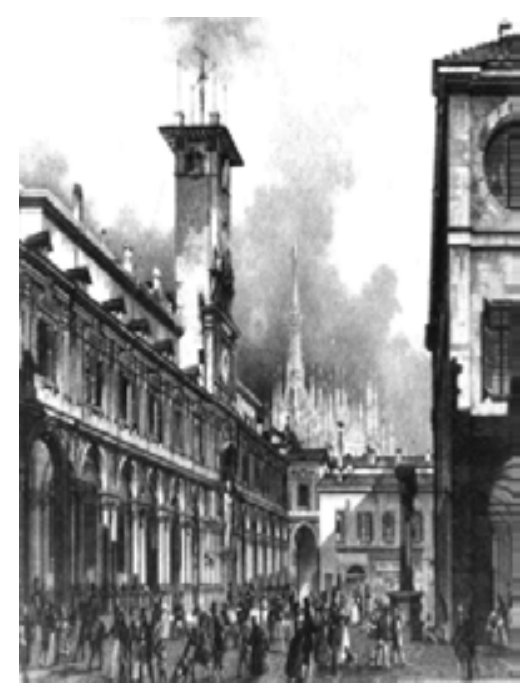

Fig. 3. Piazza dei Tribunali, sullo sfondo i locali comunali in cui ebbe la sua prima sede (1841-1866) la Società d'Incoraggiamento d'Arti e Mestieri.

\section{GABINETT0 \\ TECNOLOGICO}

DELE. L It. ISTITUTO LOMBAROV

in sacke, uTme ib un

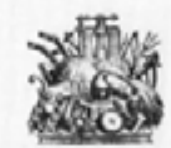

MII. 1 O

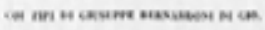

1846

Fig. 4. Gabinetto tecnologico dell'I. R. Istituto Lombardo di scienze, lettere ed arti, Milano, 1846. 


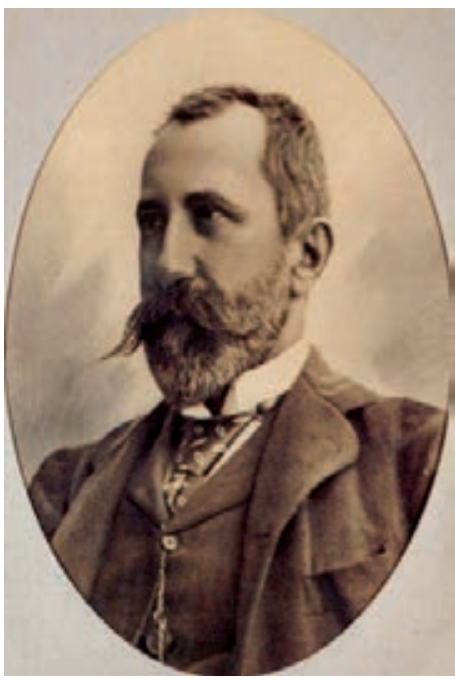

Fig. 5. Giuseppe Colombo (1836-1921).

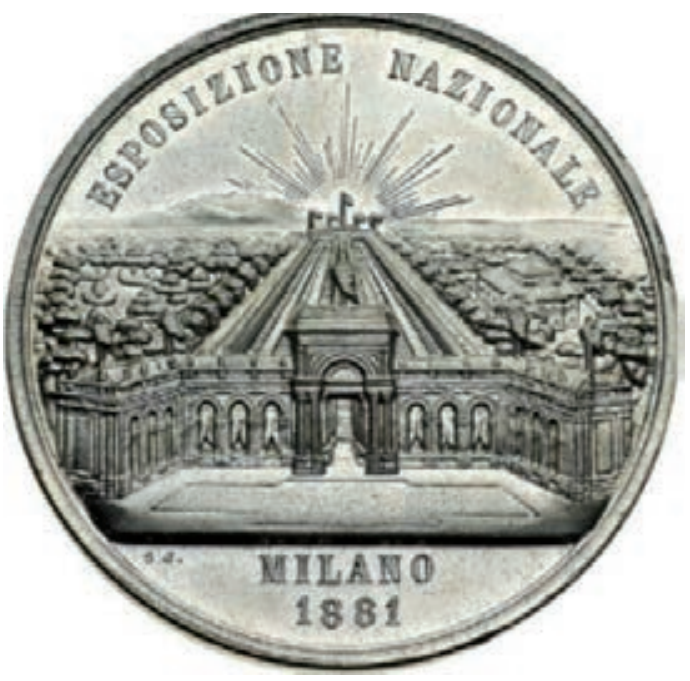

Fig. 6. Medaglione dell'Esposizione Nazionale di Milano 1881. 


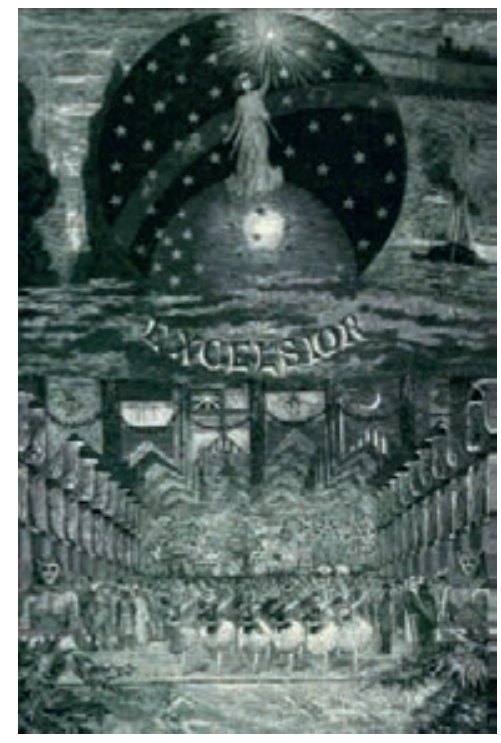

Fig. 7. Raffigurazione del Ballo Excelsior, coreografia di Luigi Manzotti, musiche di Romualdo Marenco, scene e costumi di Alfred Ende.

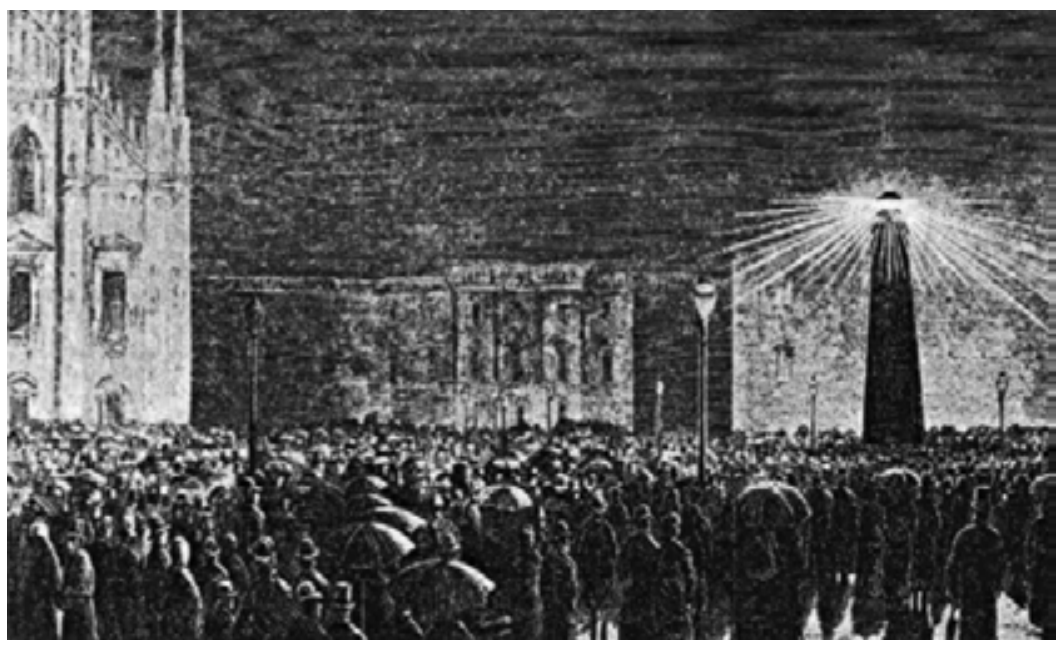

Fig. 8. Piazza del Duomo illuminata da cinque lampade ad arco voltaico Serrin alimentate da una dinamo Cabella (1876). 


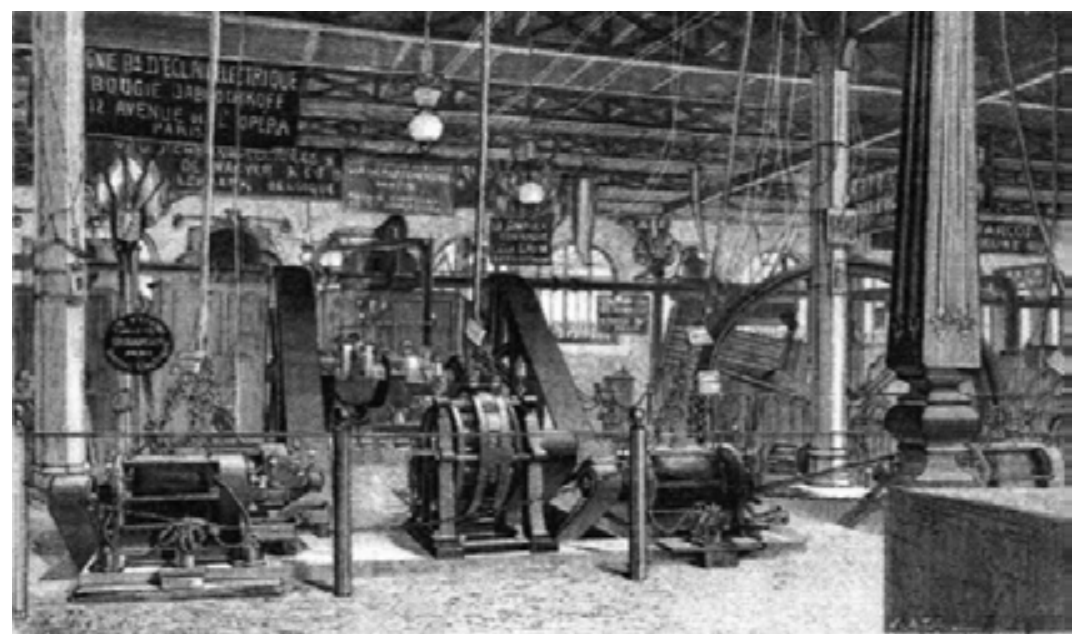

Fig. 9. Exposition internationale d'Électricité Paris, 1881.

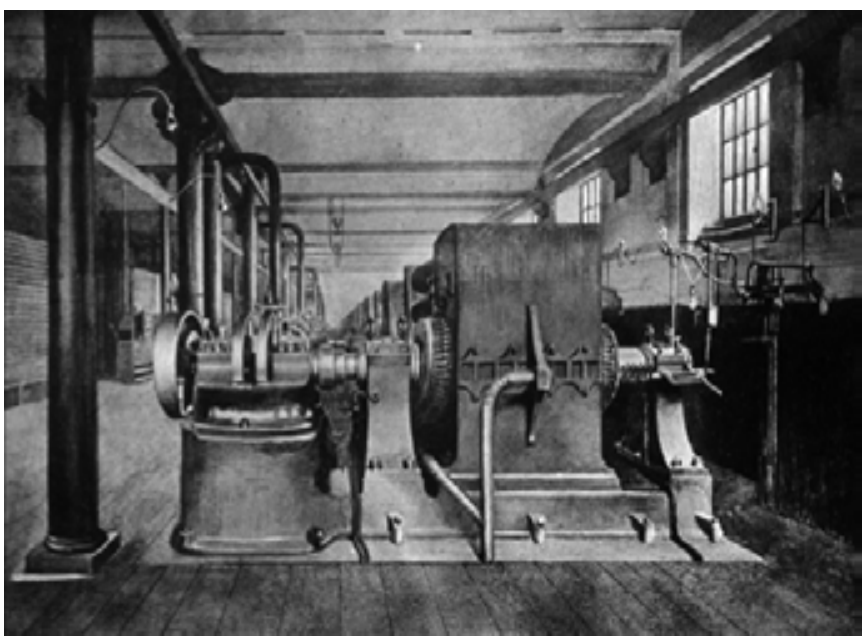

Fig. 10. Centrale termoelettrica di Santa Radegonda, 1883. 


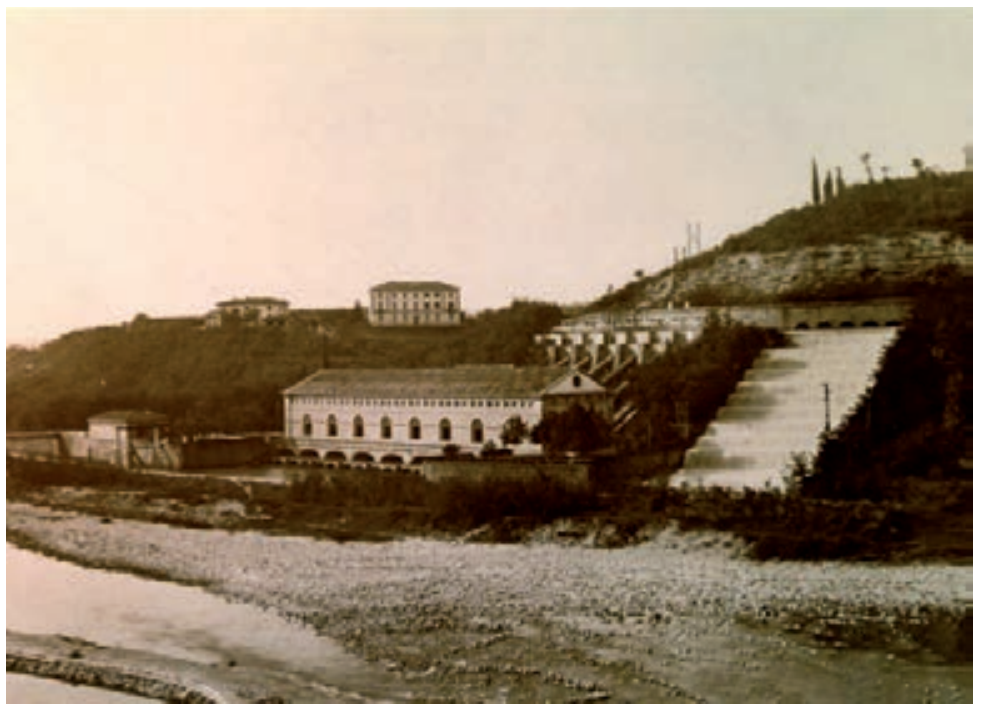

Fig. 11. Centrale idroelettrica Angelo Bertini, Paderno d'Adda, 1898.

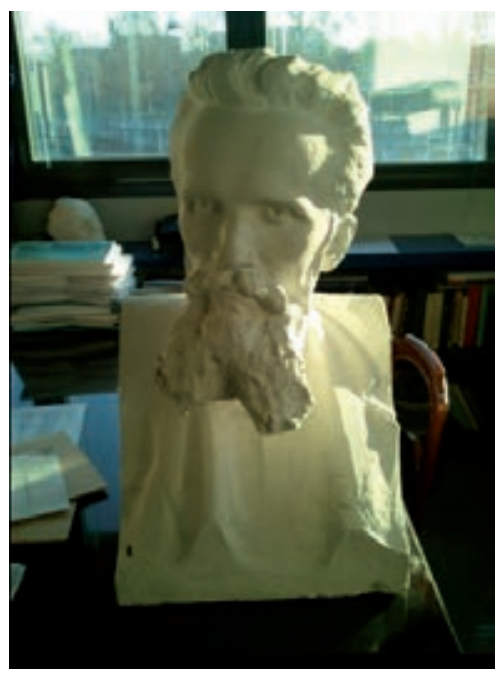

Fig. 12. Busto in gesso di Galileo Ferraris (1847-1897), Archivio Galileo Ferraris, Politecnico di Milano. 


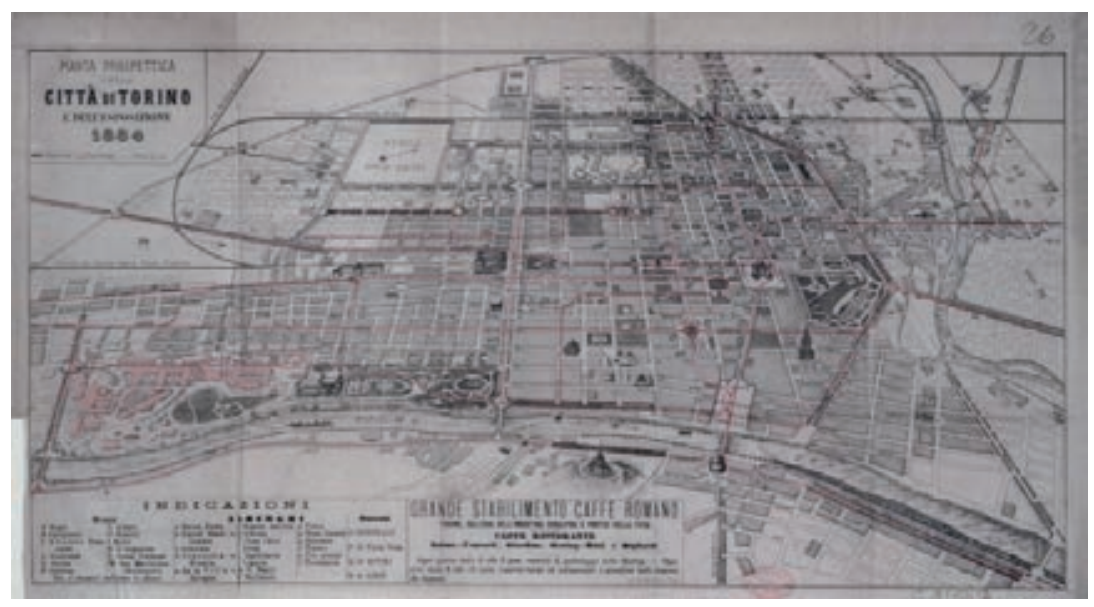

Fig. 13. Pianta prospettica della Mostra internazionale di Elettricità di Torino (1884).

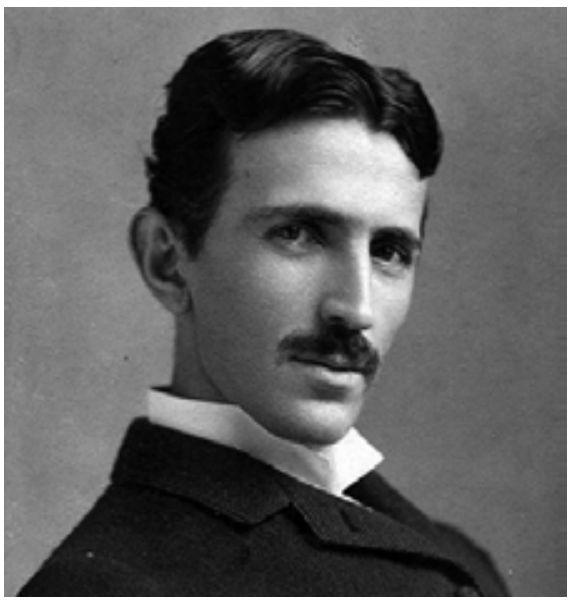

Fig. 14. Nikola Tesla (1856-1943). 


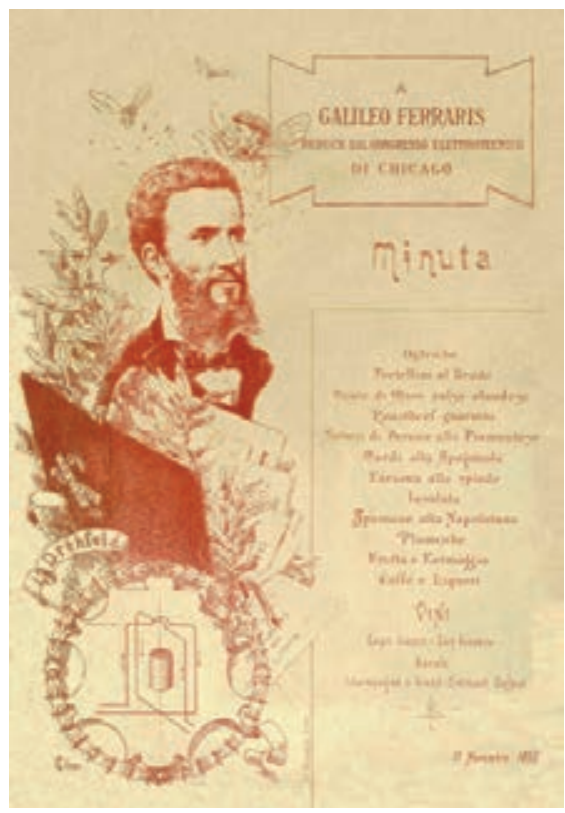

Fig. 15. Menu in onore di Galileo Ferraris, reduce dal Congresso Elettrotecnico di Chicago (1893).

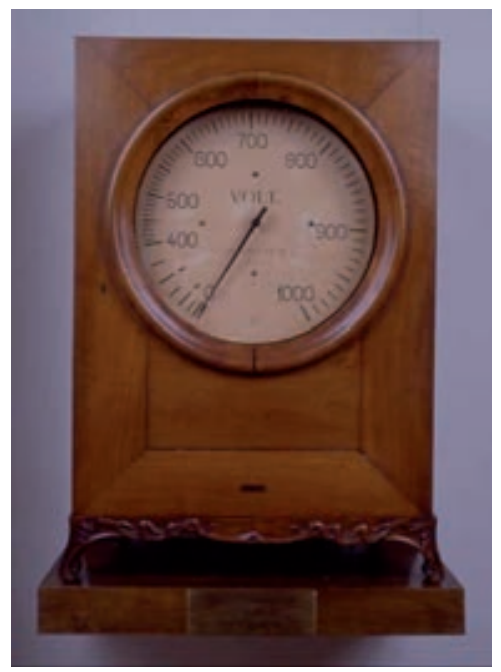

Fig. 16. Voltmetro della ditta Centimetro Grammo Secondo (CGS), già nel Dipartimento di Elettrotecnica del Politecnico di Milano. 


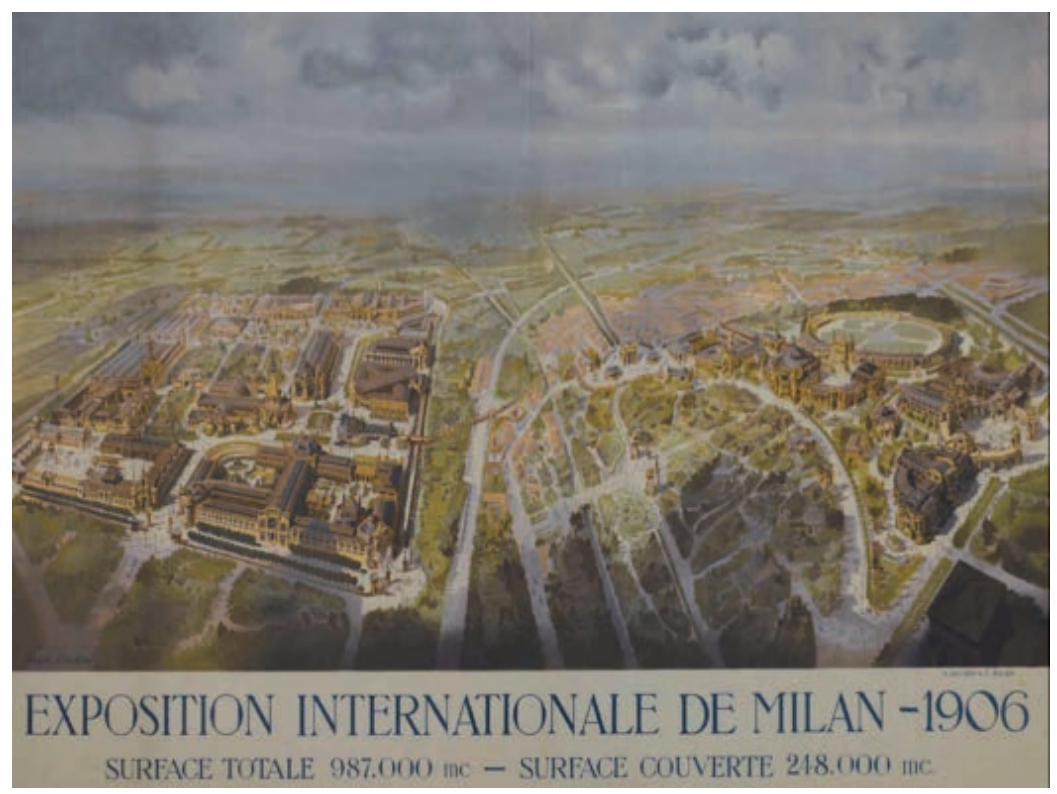

Fig. 17. Esposizione internazionale di Milano, 1906.

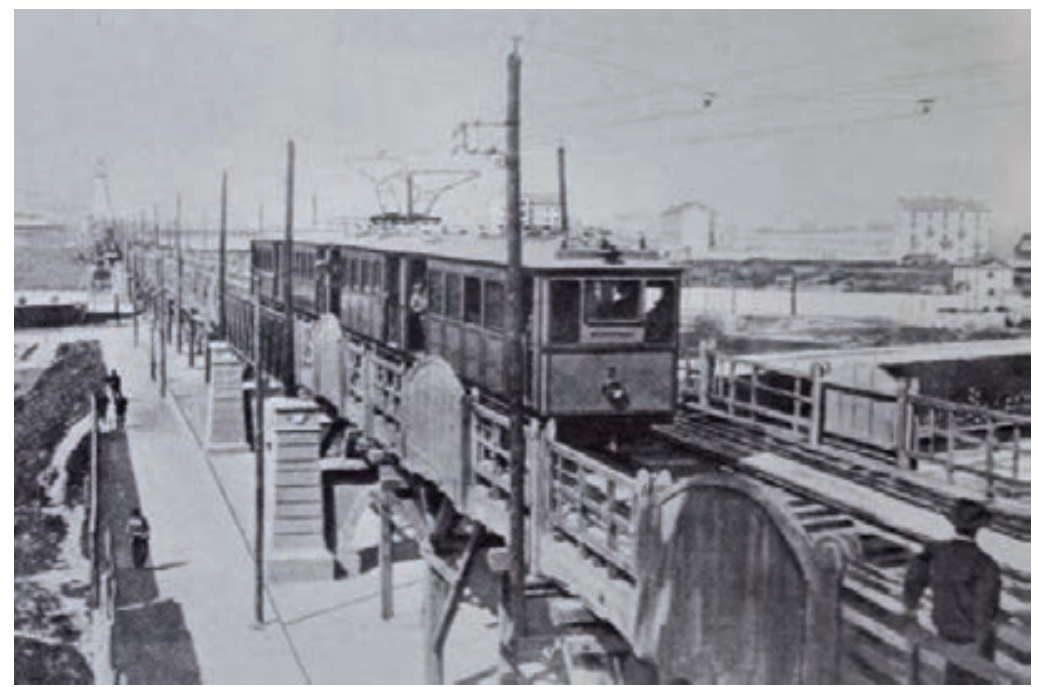

Fig. 18. Ferrovia sopraelevata realizzata per collegare le due aree in cui si teneva l'Esposizione universale del 1906 a Milano: il parco Sempione e la piazza d'Armi. 


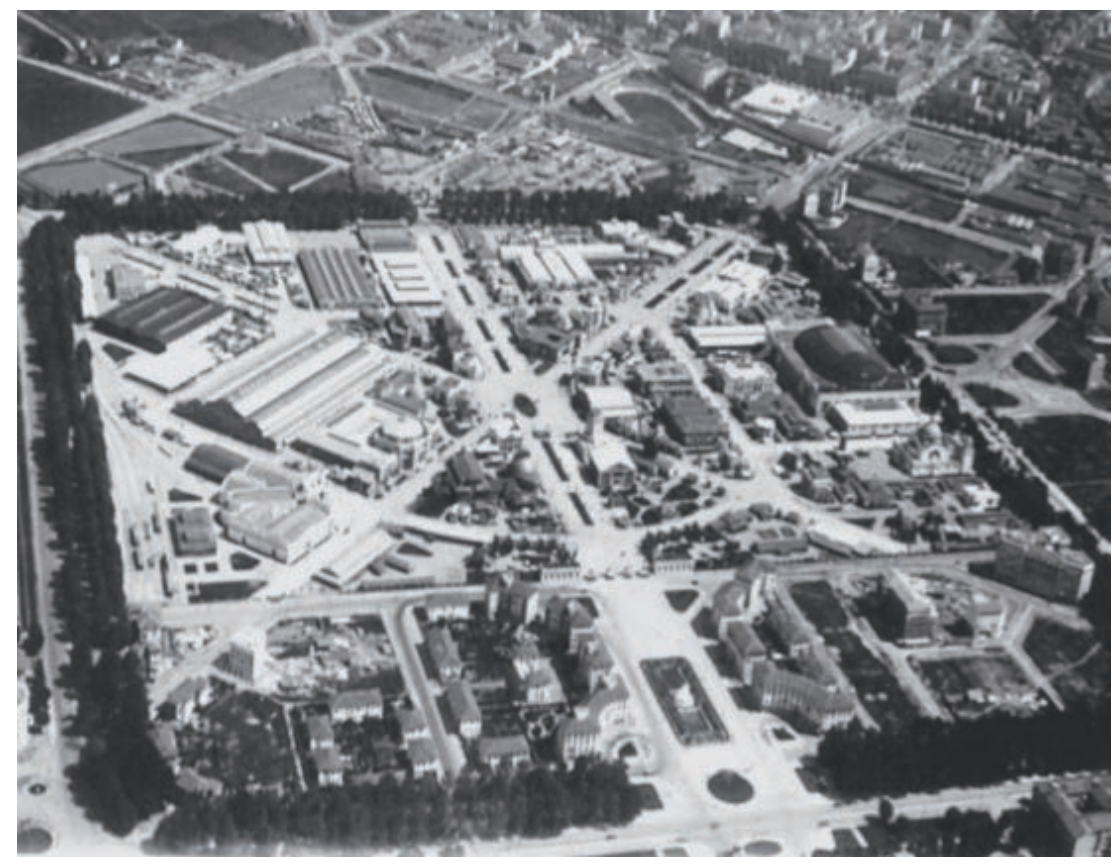

Fig. 19. Fotografia aerea della Fiera di Milano, anni '20. 